\title{
Variations in popliteal fossa venous anatomy: implications for diagnosis of deep-vein thrombosis
}

\author{
A. Sadowska1 , J.H. Spodnik², S. Wójcik² \\ 'Student Research Group of the Department of Anatomy and Neurobiology, Medical University of Gdansk, \\ Gdansk, Poland \\ 2Department of Anatomy and Neurobiology, Medical University of Gdansk, Gdansk, Poland
}

[Received 23 October 2012; Accepted 28 October 2012]

\begin{abstract}
Background: To retrospectively review the bilateral venous system within the popliteal fossa to evaluate the types of variations and their frequency seen in venous anatomy.

Materials and methods: During routine dissection of formalin-fixed cadavers, a retrospective review of 32 bilateral (64 limbs) lower limbs obtained from adult donors was performed. Deep veins present in the popliteal fossa were evaluated according to predetermined criteria for the presence of duplication of vessels and interindividual variations in venous anatomy.

Results: More than one deep venous vessel was seen in the popliteal fossa in 20 (31.3\%) of 64 limbs. In 12 (18.7\%) cases there was a high (just below the level of the adductor hiatus) origin of the popliteal vein: from 2 tributaries in $10(15.6 \%)$ and 3 tributaries in $2(3.1 \%)$. In 5 (7.8\%) cases true duplicated popliteal veins were observed. There were also $3(4.7 \%)$ cases, including one bilateral, of persistent sciatic vein.

Conclusions: Variations in popliteal fossa venous anatomy are common and have important implications for the diagnosis of deep vein thrombosis. (Folia Morphol 2013; 72, 1: 51-56)
\end{abstract}

Key words: popliteal vein, sciatic vein, anatomy variations

\section{INTRODUCTION}

Venous anatomy is known to be highly variable. In the lower limbs the most commonly present are two systems collecting blood: deep and superficial. They are separated by the fascia surrounding the thigh muscles, which defines the two compartments of the thigh: the superficial compartment, which consists of the subcutaneous tissues between the skin and the muscular fascia; and the deep compartment, which includes all tissues deep to the fascia. Blood from the superficial compartment is drained by the superficial and "perforating veins", blood from the deep compartment, by the deep veins. The short veins pierce the fascia and interconnect both systems. According to the new proposal of surgical terminology [13] they are named "perforating veins". This might lead to miscommunications because, according to the official anatomic nomenclature, the tributaries to the deep femoral vein are also named perforating veins.

According to the information from most established anatomy textbooks, the deep venous system of the lower limb is described as consisting of a continuous flow of veins. Up to the level of the calf they are usually paired [17], and from the level of the

Address for correspondence: S. Wójcik, MD, PhD, Department of Anatomy and Neurobiology, Medical University of Gdansk, ul. Dębinki 1, 80-210 Gdańsk, Poland, tel: +48 5834914 02, fax: +48 5834914 21, e-mail: swoj@gumed.edu.pl 

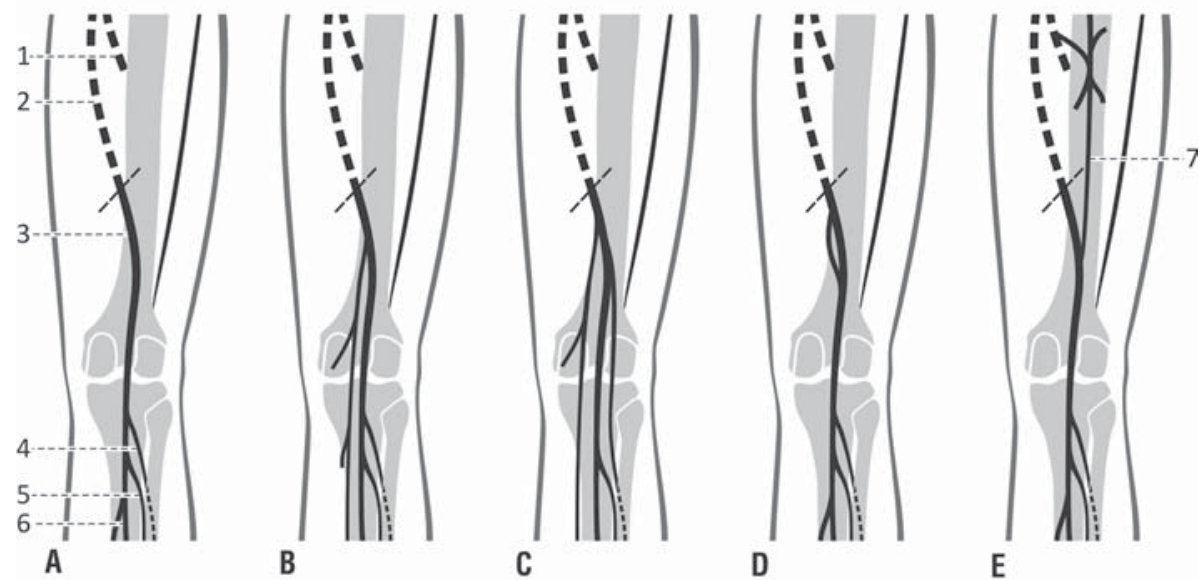

Figure 1. Patterns of deep venous system distribution within popliteal fossa and variations in the formation of popliteal vein; $\mathbf{A}$. Single popliteal vein within the popliteal fossa; B. High origin of the popliteal vein from two tributaries; C. High origin of the popliteal vein from three tributaries; D. True duplication of the popliteal vein; E. Persistent sciatic vein accompanying and anastomosing with the popliteal vein. Posterior view of the right lower limb. Oblique dashed line represents adductor hiatus; 1 — deep femoral vein; 2 - femoral vein; 3 - popliteal vein; 4 - anterior tibial vein; 5 - fibular vein; 6 - posterior tibial vein; 7 - persistent sciatic vein.

knee joint the main venous trunks (popliteal, femoral, and deep femoral) are without duplication. Numerous variations in the anatomy of the lower limb venous system have been found with the use of cadavers, ultrasonography, and venography [17], and it was demonstrated that only $17 \%$ of patients will have this present in the textbooks about "normal" venous anatomy [5]. Although the most common variation was reported in the femoral vein where, according to some previous study findings, even more than $45 \%$ of patients have variations in form of duplicated or multiple vessels, the next most common variation is related to the popliteal vein [11, 17, 21].

It is important to know not only the normal anatomy but also the variations of veins of the lower limb in detail since deep-vein thrombosis primarily occurs in this part of the body [8]. Severe complications of deep-vein thrombosis, as a postphlebitic syndrome and pulmonary embolism, are common conditions leading to death, which makes this disorder even more important. Due to the fact that the current techniques $[8,20]$ used for the diagnosis of deep venous thrombosis are based on knowing facts about anatomy, our thought is that there is a need for an extensive review of the venous anatomy of the lower limb. Therefore, the purpose of our study was to retrospectively review the bilateral venous system within the popliteal fossa to evaluate the types of variations and their frequency seen in venous anatomy.

\section{MATERIALS AND METHODS}

The dissection of 32 bilateral lower limbs (64 limbs) obtained from cadavers of adult donors ( 15 male and 17 female) of Caucasian race, who lived in the Pomerania region of Poland, was performed. Cadavers preserved in formaldehyde solution were dissected at the laboratory of the Department of Anatomy and Neurobiology, Medical University of Gdansk, Poland during the academic years 2009-2012. Deep veins in the popliteal fossa were evaluated according to predetermined criteria for the presence of duplication of vessels and interindividual variations in venous anatomy. The nomenclature used in this manuscript describing the veins of the lower limb was in accordance with those proposed by International Union and Angiology [2]. Measurement of the popliteal vein diameter was performed just below the adductor hiatus.

\section{RESULTS}

Out of 64 analysed lower limbs, 44 (68.7\%) presented a classical pattern of deep venous system distribution with a single popliteal vein within the popliteal fossa (Fig. 1A). Among the 34 lower limbs from female cadavers, a single popliteal vein was found in $64.7 \%$; while among the 30 lower limbs from male cadavers, it was $70 \%$. The diameter of the popliteal vein in females varied from 5 to $12 \mathrm{~mm}$, while in males it was from 7 to $13 \mathrm{~mm}$. The percentage distribution of popliteal vein, according to its diameter, in studied groups is shown on the Figure 2 . 


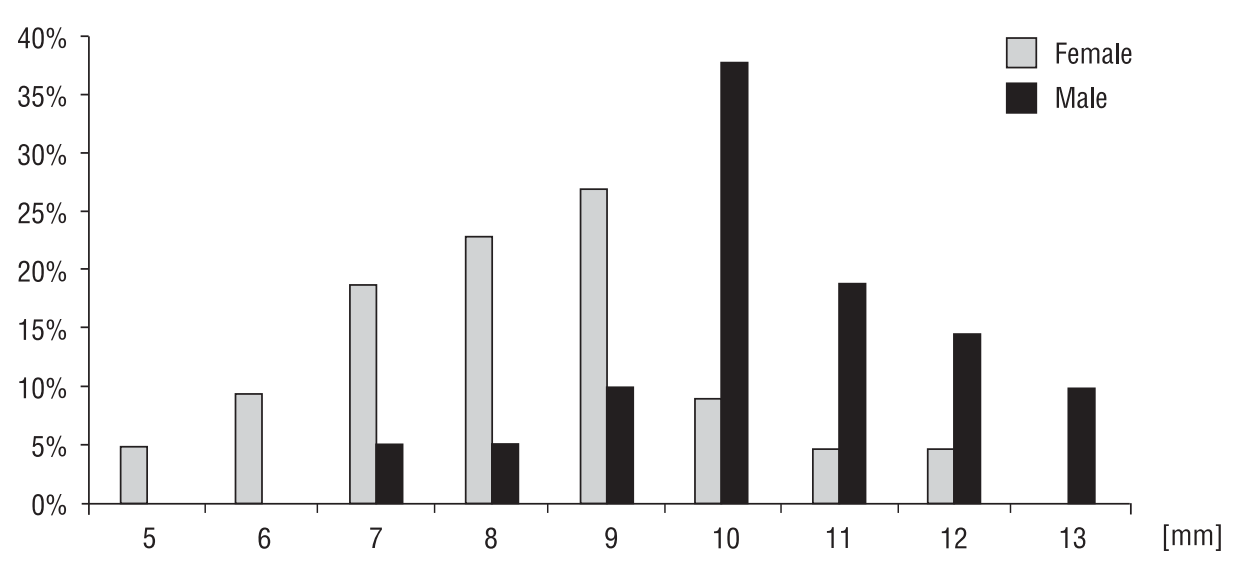

Figure 2. Percentage distribution of popliteal vein, according to its diameter, in studied groups.

More than one deep venous vessel was found in the popliteal fossa on 20 (31.3\%) of 64 limbs, 11 in females and 9 in males (Fig. 1B-E). In 12 lower limbs $(18.7 \%)$ there was a high (just below the level of the adductor hiatus) origin of the popliteal vein (Fig. 1B, C). Out of these, the high origin popliteal vein was made from two tributaries in 10 (15.6\%) and three tributaries in $2(3.1 \%)$. When two tributaries of the popliteal vein were present (6 cases in females and 4 cases in males), the larger one (diameter range from 6 to $15 \mathrm{~mm}$ ) was made by the union of the anterior tibial vein and the posterior tibial vein receiving blood from the fibular vein, and the smaller one (range of the diameter from 1 to $6 \mathrm{~mm}$ ) was formed by the other posterior tibial vein and a strong muscular branch from the gastrocnemius muscle. The diameter of the popliteal vein formed from two trunks was from 6 to $15 \mathrm{~mm}$. When three tributaries of the popliteal vein were present (1 case in females and 1 case in males), the biggest one was made by the posterior tibial vein receiving blood from the fibular vein, the middle one was made by the other posterior tibial vein and a strong muscular branch from the gastrocnemius muscle, and the smallest one was the anterior tibial vein. The diameter of the popliteal veins formed from three trunks were 9 and $14 \mathrm{~mm}$.

In 5 lower limbs (7.8\%) true duplicated popliteal veins were present (Fig. 1D, Fig. 3A). Four of this variation were observed in female lower limbs and one in male. The diameters of duplicate vessels were 5 to $9 \mathrm{~mm}$ in bigger one, and 2 to $7 \mathrm{~mm}$ in the smaller ones, their length was $80 \pm 5 \mathrm{~mm}$, and the diameter of popliteal vein formed from by its two vessels varied from 9 to $10 \mathrm{~mm}$. Duplicated popliteal veins were observed either anteriorly or posteriorly to the popliteal artery.

The most rare finding was presence within the popliteal fossa in $3(4.7 \%)$ cases of a large deep vein that was ascending into the posterior aspect of the thigh (Figs. 1E, 3B). This vessel was observed to be bilateral in one female and unilateral in one male, and we recognized it as a persistent sciatic vein (PSV). In the female this vessel on the left side was $265 \mathrm{~mm}$ long and was formed as an effect of bifurcation of the popliteal vein. It was located within the posterior compartment of the thigh muscles. It ran over the adductor magnus muscle between the biceps femoris and semimembranous and semitendinous muscles, accompanying the tibial nerve (due to the presence of early proximal division of the sciatic nerve at the level of the quadrates femoris into the tibial and common peroneal nerves). It emptied into the first perforating vein from where the deep femoral vein crossed inferior margin of quadratus femoris. It received muscular tributaries from the adductor magnus, biceps femoris, semitendinous, semimembranous, gluteus maximus, and quadratus femoris muscles. The diameter of the popliteal vein before bifurcation was $9 \mathrm{~mm}$, the diameter of the left PSV was $8 \mathrm{~mm}$, and and rest of the popliteal vein was $8 \mathrm{~mm}$. The diameters of the left femoral vein and left deep femoral vein were 12 and $8 \mathrm{~mm}$, respectively. The last two results did not differ from corresponding parameters obtained by us from other female lower limbs. The right PSV was $320 \mathrm{~mm}$ long and its distal part started from the popliteal vein. The diameter of the right popliteal vein before bifurcation was $9 \mathrm{~mm}$, the diameter of the right PSV was $6 \mathrm{~mm}$, and the remaining part of the popliteal 

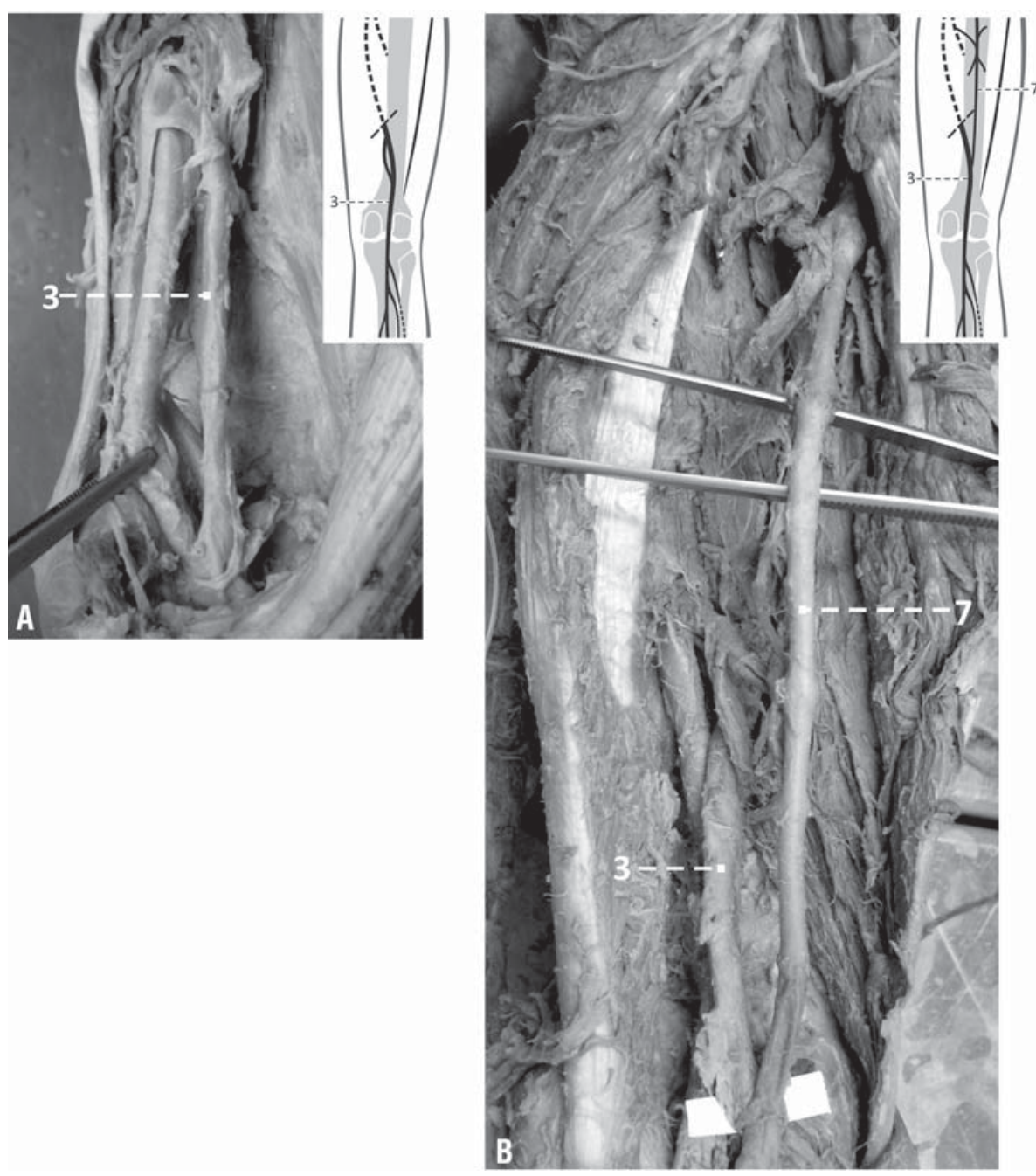

Figure 3. Microphotographs of variations in the patterns of deep venous system distribution within the popliteal fossa. Posterior view of right lower limbs; A. True duplication of the popliteal vein (3); B. Anastomosis between right popliteal vein (3) and right persistent sciatic vein (7). Numbers and diagrams correspond to the Figure 1.

vein was $8 \mathrm{~mm}$. The diameters of the right femoral vein and the right deep femoral vein were 14 and $9 \mathrm{~mm}$, respectively. The last two results did not differ from corresponding parameters obtained by us from other female lower limbs. The right PSV was located within the posterior compartment of the thigh muscles. It ran over the adductor magnus muscle between the biceps femoris and semimembranous and semitendinous muscles, accompanying the sciatic nerve. It emptied into the first perforating vein from where the deep femoral vein crosses the inferior margin of the quadratus femoris. It received blood from the adductor magnus, biceps femoris, semitendinous, semimembranous, gluteus maximus, and quadratus femoris muscles. In the third casethe PSV present on the male left lower limb was $175 \mathrm{~mm}$ long and was formed as an effect of bifurca- tion of the popliteal vein. It was located within the posterior compartment of the thigh muscles. It ran over the adductor magnus muscle between the biceps femoris and semimembranous and semitendinous muscles, posteriorly to the sciatic nerve. It emptied into the first perforating vein from where the deep femoral vein crossed the inferior margin of the quadratus femoris. It received muscular tributaries from the adductor magnus, hamstring muscles, gluteus maximus, and quadratus femoris muscles. The diameter of the popliteal vein before bifurcation was $9 \mathrm{~mm}$, the diameter of left PSV was $5 \mathrm{~mm}$, and the rest of the popliteal vein was $9 \mathrm{~mm}$. The diameters of the left femoral vein and the left deep femoral vein were 12 and $9 \mathrm{~mm}$, respectively. The last two results did not differ from corresponding parameters obtained by us from other male lower limbs. 


\section{DISCUSSION}

In the present study we have shown that the variation of the deep venous vessels in the popliteal fossa are present in more than $30 \%$ of studied lower limbs. In approximately $19 \%$ of the studied lower limbs there was a high (just below the level of the adductor hiatus) origin of the popliteal vein formed by two or three tributaries. In almost $8 \%$ of the studied cases we found true duplicated popliteal veins, while persistent sciatic vein was present in about $5 \%$ of cases.

True duplicated popliteal veins are not a very common finding, but their presence is considered as a risk factor for deep-vein thrombosis. Similar to our data were the results of a previous study based on a retrospective review of bilateral venograms free of thrombus [17], in which the authors showed the incidence of true duplicated popliteal veins in $5.1 \%$ of studied lower limbs. In a retrospective study performed to determine the prevalence of lower extremity venous duplication using duplex ultrasound [6], duplication of the popliteal vein was observed in $3.6 \%$ of cases, while in more recent studies performed with the same technique [21] it was shown to be even more rare: from $0.9 \%$ during unilateral studies to $0.6 \%$ during bilateral studies. Probably the small number of this popliteal vein variation is connected with the fact that there are only a limited number of reports stressing the presence of the thrombus in one of two veins in patients with true duplication of the popliteal vein $[1,7]$.

Although the presence of true duplication of the popliteal vein described above is quite rare, a high origin of the popliteal vein from two or more tributaries is much more common. In our material this anatomical variant was present in almost $19 \%$ of studied lower limbs. The study mentioned above [17], based on a retrospective review of bilateral venograms, showed the presence of two or three deep venous tributaries of the popliteal vein within popliteal fossa in $44.1 \%$ of the studied lower limbs. A significantly higher percentage, almost $60 \%$, of high union tributaries of the popliteal vein was reported on the basis of a retrospective review of computed tomography venography images [15] obtained from the Korean population. What is really striking in this last group of patients is that $99 \%$ of unions were due to the high union of the anterior tibial vein with the popliteal vein. On the contrary, in our material, instead of the anterior tibial vein, one of the posterior tibial veins participated in this high union with the popliteal vein. All those studies indicate the high incidence of multiple vessels in the popliteal fossa, rather high origin of the popliteal vein, but not its true duplication, which is significant for imaging this region during diagnosis of deep-vein thrombosis $[8,20]$. The presence of numerous variations among deep veins within the popliteal fossa raises the risk of the potential for deep-vein thrombosis formation secondary to changes in flow velocities. This may also amplify the possibility of a false negative result during deep-vein thrombosis diagnostic procedures in cases of a missed thrombus during ultrasonography image analysis, if only a single vein is visible.

The sciatic vein serves as the most important collector of blood from the lower limb during the prenatal period. Persistent sciatic vein is considered a rare congenital anomaly [4]. As observed by us, the $5 \%$ frequency of this anomaly corresponds with recent results of $6.25 \%$ obtained during analysis of lower limbs from 16 cadavers [3].

Persistent sciatic vein may communicate with the terminal part of the popliteal vein or the small saphenous vein, and quite often anastomoses with the medial circumflex femoral vein or, as in our cases, with one of the perforating veins (tributaries of the deep femoral vein). According to the classification proposed by Cherry et al. [4], persistent sciatic vein may be classified in three variants: complete, proximal, or superior and distal or inferior. Taking into account that all three persistent sciatic veins observed by us have their origin in the popliteal vein and were found in the medial portion of the thigh and were drained via the first perforating vein into deep femoral vein, we consider them to be a distal variant of persistent sciatic vein. Persistent sciatic artery is an even more uncommon congenital malformation than persistent sciatic vein. Its incidence is estimated to be $0.025-0.04 \%$ [19]. Although embryology suggests the presence of persistent sciatic vein may occur together with persistent sciatic artery, which has been reported [16, 22], in our material we did not find persistent sciatic artery. However, similarly to this report, we observed early proximal division of the sciatic nerve into the tibial and common peroneal nerves.

The deep femoral vein provides an important collateral pathway for blood drainage when the femoral vein is greatly obstructed or occluded by thrombosis. In such conditions phlebography gives a great deal of challenging data relating to collateral blood flow [18]. It is believed that the deep femoral-popliteal collateral connections may have an embryological basis. The size of this connection depends on the degree of obstruction present in the femoral vein. In extreme cases the deep femoral vein completely replaces the femo- 
ral vein as the main outflow from the limb. This axial transformation of the deep femoral vein can be strikingly similar to normal radiographic anatomy, with smooth contours that may be mistaken by the unwary for the femoral vein during $X$-ray examination. Once formed, the deep femoral-popliteal connector persists and does not disappear even when the superficial femoral vein has recanalised satisfactorily. In our material we observed the bilateral presence of persistent sciatic vein rather than the abovedescribed transformation. According to previously published data, axial transformation of the deep femoral vein leads to diminished size of the femoral vein [18], which was not observed by us. Also, the typical pathway of vessels being an effect of axial transformation is different. In fact, venous anastomoses run along the femur, within the adductor compartment [14], not close to the hamstring muscle as was observed in our material. It also has to be mentioned that persistent sciatic vein was described as unusual but important: location of varicose veins $[10,16]$ and thromboses [12] as well as unusual causes of reflux from the popliteal fossa and sural nerve damage [9].

The present study confirms that variations in popliteal fossa venous anatomy are common, which may have important implications for the diagnosis of deep vein thrombosis.

\section{ACKNOWLEDGEMENTS}

The authors wish to thank to Ms Sylwia Scisłowska, MA, for the preparation of illustrations.

\section{REFERENCES}

1. Bronzi G, Venarucci V, Delle Monache G, Scudieri M, Bellagamba G (1989) Thrombosis of congenital double popliteal vein: case report. Angiology, 40: 933-936.

2. Caggiati A, Bergan JJ, Gloviczki P, Eklof B, Allegra C, Partsch H (2005) Nomenclature of the veins of the lower limb: extensions, refinements, and clinical application. J Vasc Surg, 41: 719-724.

3. Cardoso BB, Alvarenga CO, Miyahara MdS, Burihan MC, Lima MRQAd, Kuwahara MC, Silva RCMe (2010) Persistęncia da veia ciática. J Vascular Brasileiro, 9: 137-140.

4. Cherry KJ, Gloviczki P, Stanson AW (1996) Persistent sciatic vein: diagnosis and treatment of a rare condition. J Vasc Surg, 23: 490-497.

5. Cockett FB (1954) Abnormalities of the deep veins of the leg. Postgrad Med J, 30: 512-522.

6. Dona E, Fletcher JP, Hughes TM, Saker K, Batiste P, Ramanathan I (2000) Duplicated popliteal and superfi- cial femoral veins: incidence and potential significance. Aust N Z J Surg, 70: 438-440.

7. Gerkin TM, Beebe HG, Williams DM, Bloom JR, Wakefield TW (1993) Popliteal vein entrapment presenting as deep venous thrombosis and chronic venous insufficiency. J Vasc Surg, 18: 760-766.

8. Goldhaber SZ, Bounameaux H (2012) Pulmonary embolism and deep vein thrombosis. Lancet, 379: 1835-1846.

9. Hamilton HE, Darke SG (1999) Persistent sciatic vein unusual cause of reflux from the popliteal fossa and sural nerve damage. Eur J Vasc Endovasc Surg, 17: 539-541.

10. Jung SC, Lee W, Chung JW, Jae HJ, Park EA, Jin KN, Shin Cl, Park JH (2009) Unusual causes of varicose veins in the lower extremities: CT venographic and Doppler US findings. Radiographics, 29: 525-536.

11. Kerr TM, Smith JM, McKenna P, Lutter KS, Sampson MG, Helmchen RH, Roedersheimer LR (1992) Venous and arterial anomalies of the lower extremities diagnosed by duplex scanning. Surg Gynecol Obstet, 175: 309-314.

12. Labropoulos N, Bekelis K, Leon LR Jr (2008) Thrombosis in unusual sites of the lower extremity veins. J Vasc Surg, 47: 1022-1027.

13. May R, Mignon G (1981) The nomenclature of the clinically most important venae perforantes. Rofo, 134 : 688-690.

14. Natsis K, Totlis T, Paraskevas G, Papathanasiou E, Sofidis G, Noussios G (2008) Axial transformation of the profunda femoris vein: formation, relations and course in a cadaveric specimen. Folia Morphol, 67: 304-306.

15. Park EA, Chung JW, Lee W, Yin YH, Ha J, Kim SJ, Park JH (2011) Three-dimensional evaluation of the anatomic variations of the femoral vein and popliteal vein in relation to the accompanying artery by using CT venography. Korean J Radiol, 12: 327-340.

16. Parry DJ, Aldoori MI, Hammond RJ, Kessel DO, Weston M, Scott DJ (2002) Persistent sciatic vessels, varicose veins, and lower limb hypertrophy: an unusual case or discrete clinical syndrome? J Vasc Surg, 36: 396-400.

17. Quinlan DJ, Alikhan R, Gishen P, Sidhu PS (2003) Variations in lower limb venous anatomy: implications for US diagnosis of deep vein thrombosis. Radiology, 228: 443-448.

18. Raju S, Fountain T, Neglen P, Devidas M (1998) Axial transformation of the profunda femoris vein. J Vasc Surg, 27: 651-659.

19. Santaolalla V, Bernabe MH, Hipola Ulecia JM, De Loyola Agundez Gomez I, Hoyos YG, Otero FJ, Mendizabal RF, Maldonado FJ, Legrand JL (2010) Persistent sciatic artery. Ann Vasc Surg, 24: e697-e610.

20. Scarvelis D, Wells PS (2006) Diagnosis and treatment of deep-vein thrombosis. CMAJ, 175: 1087-1092.

21. Simpson WL, Krakowsi DM (2010) Prevalence of lower extremity venous duplication. Indian J Radiol Imaging, 20: 230-234.

22. Tohno Y, Tohno S, Watanabe T, Watanabe M, Kimura $Y$ (1993) Anomaly of bilateral persistent sciatic arteries. Kaibogaku Zasshi, 68: 422-428. 